\title{
Vsx1 Regulates Terminal Differentiation of Type 7 ON Bipolar Cells
}

\author{
Zhiwei Shi, ${ }^{1 \star}$ Stuart Trenholm, ${ }^{2 *}$ Minyan Zhu, ${ }^{1}$ Sarah Buddingh, ${ }^{1}$ Erin N. Star, ${ }^{1}$ Gautam B. Awatramani, ${ }^{2}$ \\ and Robert L. Chow ${ }^{1}$ \\ ${ }^{1}$ Department of Biology, University of Victoria, Victoria, British Columbia V8W 3N5, Canada, and 2Department of Anatomy and Neurobiology, Dalhousie \\ University, Halifax, Nova Scotia B3H 1X5, Canada
}

\begin{abstract}
Although retinal bipolar cells represent a morphologically well defined population of retinal interneurons, very little is known about the developmental mechanisms that regulate their processing. Furthermore, the identity of specific bipolar cell types that function in distinct visual circuits remains poorly understood. Here, we show that the homeobox gene Vsx 1 is expressed in Type 7 ON bipolar cells. In the absence of $V s x 1$, Type 7 bipolar cells exhibit proper morphological specification but show defects in terminal gene expression. Vsxl is required for the repression of bipolar cell-specific markers, including Calcium-binding protein 5 and Chx10. This contrasts its genetic requirement as an activator of gene expression in OFF bipolar cells. To assess possible ON signaling defects in $V$ s $x 1$-null mice, we recorded specifically from ON-OFF directionally selective ganglion cells (DSGCs), which cofasciculate with Type 7 bipolar cell terminals. Vsx1-null ON-OFF DSGCs received more sustained excitatory synaptic input, possibly due to Type 7 bipolar cell defects. Interestingly, in Vsx1-null mice, the directionally selective circuit is functional but compromised. Together, these findings indicate that $V s x 1$ regulates terminal gene expression in Type 7 bipolar cells and is necessary for proper $\mathrm{ON}$ visual signaling within a directionally selective circuit.
\end{abstract}

\section{Introduction}

Retinal bipolar cells, the interneurons linking photoreceptors to ganglion cells, have been anatomically well characterized. In the mammalian retina, $\sim 10-12$ morphologically and molecularly unique cell types have been described, each likely serving a distinct physiological role (Masland, 2001; Ghosh et al., 2004; Wässle et al., 2009). However, understanding the mechanisms that regulate the functional development of specific bipolar cell types has proven difficult.

Recently, cell type-specific markers have aided in the identification of transcription factors that regulate unique aspects of retinal development and function. Homeodomain transcription factors, along with the basic helix-loop-helix transcription factors, represent excellent candidates as specific regulators of retinal development. Importantly, such transcription factors are required for retinal bipolar cell type differentiation and homeostasis (Bramblett et al., 2004; Chow et al., 2004; Ohtoshi et al., 2004; Cheng et al., 2005; Feng et al.,

\footnotetext{
Received May 10, 2011; revised June 20, 2011; accepted June 29, 2011.

Author contributions: R.L.C., Z.S., S.T., and G.B.A. designed research; Z.S., S.T., M.Z., S.B., and R.L.C. performed research; E.N.S. contributed unpublished reagents/analytic tools; Z.S., S.T., G.B.A., and R.L.C. analyzed data; Z.S., S.T., G.B.A., and R.L.C. wrote the paper.

This work was supported by operating grants from the Canadian Institutes for Health Research and Foundation Fighting Blindness (R.L.C. and G.B.A.) and was undertaken, in part, thanks to funding to R.L.C. from the Canada Research Chairs program. Z.S. was supported, in part, by a bursary from the Margaret Adamson Estate. We are grateful to R. Margolskee for generously providing the GUS8.4GFP transgenic mice. We thank the following for their generosity in providing the following antibodies: F. Haeseleer (Cabp5) and Y.-Q. Ding (NK3R). We thank A. Johnson at the University of British Columbia Flow Cytometry Facility for technical assistance. We thank R. O. Wong and K.R. Delaney for helpful suggestions and comments on the manuscript.

* Z.S. and S.T. contributed equally to this work.

Correspondence should be addressed to Robert L. Chow, Department of Biology, University of Victoria, PO Box 3020, Station CSC, Victoria, BC V8W 3N5, Canada. E-mail: bobchow@uvic.ca.

DOI:10.1523/JNEUROSCI.2331-11.2011

Copyright $\odot 2011$ the authors $\quad 0270-6474 / 11 / 3113118-10 \$ 15.00 / 0$
}

2006; Elshatory and Gan, 2008; Kerschensteiner et al., 2008). Among these, the paired-like homeodomain transcription factor Vsx1 is essential for the terminal differentiation of a subset of OFF bipolar cells (Chow et al., 2004; Ohtoshi et al., 2004). Retinal cell specification and gross morphogenesis is normal in Vsxl-null mice, but there is reduced expression of at least four OFF cone bipolar cell-specific markers (Chow et al., 2004; Ohtoshi et al., 2004). A role for Vsxl in OFF bipolar cell visual signaling is supported by the finding of reduced OFF ganglion cell responses in the Vsx1-null mutant (Chow et al., 2004; Kerschensteiner et al., 2008). Vsxl is also expressed in ON bipolar cells, however, due to a lack of ON cone bipolar cell typespecific markers and the absence of an overt $\mathrm{ON}$ pathway signaling defect in Vsxl-null mice, the role of Vsxl in ON bipolar cells has remained unclear (Chow et al., 2004).

Here, we used GUS8.4GFP transgenic reporter mice (Huang et al., 1999, 2003) to show that Vsxl is expressed in Type 7 ON bipolar cells. In Vsx1-null mice, Type 7 bipolar cells are morphologically specified but exhibit defects in terminal differentiation, including an upregulation of the bipolar cell-specific proteins Chx10 and $\mathrm{Ca}^{2+}$-binding protein 5 (Cabp5), which are known to be important for bipolar cell development (Burmeister et al., 1996) and $\mathrm{Ca}^{2+}$-dependent synaptic function (Rieke et al., 2008), respectively. To assess the functional consequence of Vsxl loss of function on the output of Type $7 \mathrm{ON}$ bipolar cells, we recorded electrophysiological responses from directionally selective (DS) ganglion cells (DSGCs) which are thought to receive synaptic input from Type 7 bipolar cells (Lin and Masland, 2005). In Vsx1-null mice, ON responses in DSGCs exhibited slowed kinetics and an impaired ability to compute direction of motion. Together, our findings demonstrate an important role for Vsxl in the terminal differentiation of Type 7 ON bipolar cells. 
Table 1. List of antibodies

\begin{tabular}{llll}
\hline Antigen & Antiserum & Source & Working dilution \\
\hline Vsx1 & Rabbit anti-Vsx1 & R.L. Chow, University of Victoria, Victoria, BC, Canada & $1: 100$ \\
Cabp5 & Rabbit anit-Cabp5 & F. Haeseleer, Department of Ophthalmology, Seattle, WA & $1: 500$ \\
$\beta$-Galactosidase & Rabbit anti- $\beta$-Gal & ICN Biomedicals (Catalog \#55976) & $1: 20,000$ \\
GFP & Goat anti-GFP & Abcam (ab6673-100 & $1: 200$ \\
NK3R & Rabbit anti-NK3R & Y.Q. Ding, Chinese Academy of Sciences, Shanghai, China & $1: 100$ \\
PKC $\alpha$ & Rabbit anti-PKC $\alpha$ & Sigma (P4334) & $1: 10,000$ \\
Calretinin & Goat anti-calretinin & Chemicon (AB1559) & $1: 2500$ \\
Chx10 & Sheep anti-Chx10 & Exalpha Biologicals (X1180P) & $1: 500$ \\
\hline
\end{tabular}

\section{Materials and Methods}

Mice

Mice of either sex carrying the $V s x 1^{\tau L a c Z}$ knock-in null mutant allele (Chow et al., 2004) were maintained on a 129 S1 genetic background (The Jackson Laboratory) and crossed to mice of either sex carrying the GUS8.4GFP trans-

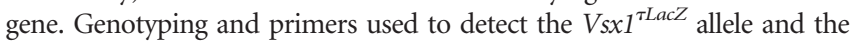
GUS8.4GFP transgene in ear punch biopsies were performed as described previously (Huang et al., 1999; Chow et al., 2004). Mice were maintained on a $12 \mathrm{~h} \mathrm{light/dark} \mathrm{cycle} \mathrm{and} \mathrm{all} \mathrm{experimental} \mathrm{procedures} \mathrm{were} \mathrm{approved} \mathrm{by} \mathrm{the}$ University of Victoria Animal Care Committee, in accordance with the Canadian Council for Animal Care.

\section{Immunolabeling}

The preparation and analysis of immunolabeled retinal sections was done on mice of either sex as previously described (Chow et al., 2001, 2004). Following removal of the cornea and lens, eyes were drop fixed in $4 \%$ paraformaldehyde in PBS for either $1 \mathrm{~h}$ on ice or $20 \mathrm{~min}$ at room temperature. After cryoprotection in 30\% sucrose overnight, eyes were embedded in Tissue-Tek O.C.T. compound (Sakura Finetek) and cryosectioned at $14 \mu \mathrm{m}$. Sections were permeabilized in PBS containing 1\% Triton X-100, blocked in PBS containing 10\% horse serum, and incubated overnight at $4{ }^{\circ} \mathrm{C}$ with primary antibodies diluted in PBS containing $1 \%$ horse serum. The primary antibodies and dilutions used are shown in Table 1. Secondary antibodies conjugated with Alexa Fluor dyes (Invitrogen) or anti-guinea pig Cy3 (Jackson Laboratories) were used at a 1:100 dilution and incubated for $1 \mathrm{~h}$ at room temperature. Images were taken on a Nikon Eclipse TE2000-U C1 confocal microscope and processed using Adobe Photoshop CS3.

\section{Quantitation of Chx10 levels in Type 7 and Type 2 retinal cone bipolar cells}

Retinal sections were triple stained and examined for immunofluorescence by using sheep anti-Chx10, chicken anti-GFP, rabbit anti-PKC $\alpha$, and rabbit anti-NK3R antibodies. Images were taken with the $60 \times$ objective as stacks of $4 \sim 5$ optical sections both above and below the brightest focal plane of Chx10 intensity with a step size of $0.45 \mu \mathrm{m}$. The brightest level of Chx10 fluorescence was acquired by setting up the photomultiplier tube gain high, but well below its saturation for each individual Z-stack. For each Chx10 labeled cell that was quantified, the maximum Chx10 intensity value within a Z-stack was used. For each individual image, Chx10 fluorescence levels in GUS8.4GFP-positive or NK3R-positive cells were normalized to Chx10 fluorescence levels in $\mathrm{PKC} \alpha$-positive rod bipolar cell. Three females were used for each genotype, and 4-10 images were examined per mouse.

\section{Flow cytometry}

Mice of either sex were euthanized by carbon dioxide and eyes were enucleated. After removal of cornea, lens, and vitreous body, retinas were rapidly dissected and separated from retinal pigmented epithelium in cold PBS. Retinal tissue was rinsed twice in PBS and then cut into 1-2 $\mathrm{mm}$ pieces. Retinal pieces were incubated in $4 \mathrm{ml}$ of papain dissociation mixture $[20 \mathrm{U} / \mathrm{ml}$ papain (Worthington Biochemical, catalog \#LS003126), $5.5 \mathrm{~mm}$ L-cysteine, $0.067 \mathrm{~mm} \beta$-mercaptoethanol, $1.1 \mathrm{~mm}$ EDTA, $100 \mathrm{U} / \mu \mathrm{l}$ DNase I, and $\mathrm{Ca}^{2+}$ - and $\mathrm{Mg}^{2+}$-free HBSS (Invitrogen)] for $12 \sim 14 \mathrm{~min}$ at $37^{\circ} \mathrm{C}$ in a water bath with gentle agitation when the pieces looked lighter and smaller. Before dissociation, the papain dissociation mix was first activated at $37^{\circ} \mathrm{C}$ for 30 min or until the papain was completely dissolved and the solution appeared clear, and then the solution was filtered using a $0.22 \mu \mathrm{m}$ membrane. The dissociation reaction was stopped by adding an equal volume of prewarmed $10 \%$ FCS/HBSS cell dissociation buffer, followed by $3 \mathrm{ml}$ of prewarmed 1\% FCS (fetal calf serum, Invitrogen, catalog \#10437036)/HBSS containing 100U/ml DNase I. The mixture was gently triturated ( $8 \sim 10$ passes) using a $5 \mathrm{ml}$ pipette and then passed through a $70 \mu \mathrm{m}$ cell strainer (BD Biosciences, BD Falcon, REF352350). The concentration of the acquired cells was determined with Bright-Line hemacytometer (Hausser Scientific). Typically, $1.6{ }^{\star} 10^{7}$ cells per 4 retinas were recovered. GUS8.4GFP-positive cells from dissociated retinas were examined on a Vantage Diva highspeed cell sorter (Becton Dickinson) and analyzed using CellQuest Pro 5.2 software (Becton Dickinson).

\section{Electrophysiology}

Retinal preparation. Whole-mount retina from mice of either sex were prepared as described previously (Borowska et al., 2011). Briefly, mice were anesthetized and decapitated. The eyes were removed and placed in warm Ringer's solution containing the following (in mM): $110 \mathrm{NaCl}, 2.5$ $\mathrm{KCl}, 1.6 \mathrm{MgCl}_{2}, 1 \mathrm{CaCl} 2,10$ dextrose, $22 \mathrm{NaHCO}_{3}$. The cornea, lens, and iris were carefully cut away, and the retina was removed from the eyecup. The isolated retina was then placed down on a $0.22 \mu \mathrm{m}$ membrane filter (Millipore) with a precut window that enabled transmitted light to reach the retina and for the preparation to be viewed under infrared illumination with the aid of a Spot RT3 CCD camera (Diagnostic Instruments) attached to an upright Olympus BX51 WI fluorescent microscope equipped with a $60 \times$ water immersion lens (Olympus Canada). The preparation was continually bathed with control Ringer's solution that was bubbled with carbogen $\left(95 \% \mathrm{O}_{2}: 5 \% \mathrm{CO}_{2} ; \mathrm{pH} 7.4\right)$. All experiments were performed near physiological temperatures $\left(35-36^{\circ} \mathrm{C}\right)$.

Light stimulus. Light stimuli were controlled using custom MATLAB/ LabVIEW software (written by Dr. David Balya; Friedrich Miescher Institute, Basel, Switzerland). Images were projected onto the retina via a DLP projector focused on the outer segments of the photoreceptors using the substage microscope condenser. The light stimulus was centered on the soma for each neuron. The preferred spot size for each cell was calculated as the peak ON and OFF spike rate obtained from a series of increasing spots $(25-800 \mu \mathrm{m})$. Directional selectivity was tested by moving a $400 \mu \mathrm{m}$ spot at $1000 \mu \mathrm{m} / \mathrm{s}$ over the cell in eight different directions equally divided over $360^{\circ}$. Directional selectivity was assessed by creating a directional selectivity index (DSI) calculated as a vector sum of the number of spikes generated for both $\mathrm{ON}$ and OFF responses in each of the eight directions, divided by the total number of spikes in all directions. The resulting vector therefore indicates the angle of the preferred direction as well as the magnitude of the asymmetry of the response. DSI closer to 1 represent stronger directional selectivity, whereas DSI closer to 0 represent more symmetrical responses.

Electrophysiological recordings. Spike recordings were obtained using the loose cell-attached patch-clamp technique using 5-10 M $\Omega$ electrodes containing Ringer's solution. Following spike recording, DSGCs were patch clamped in the whole-cell voltage-clamp mode. Whole-cell recordings were made using 4-6 $\mathrm{M} \Omega$ electrodes containing the following (in $\mathrm{mm}$ ): $112.5 \mathrm{CsCH}_{3} \mathrm{SO}_{3}, 9.7 \mathrm{KCl}, 1 \mathrm{MgCl}_{2}, 1.5 \mathrm{EGTA}, 10 \mathrm{HEPES}, 4$ ATP $\mathrm{Mg}_{2}, 0.5 \mathrm{GTP} \mathrm{Na}_{3}$, and 0.02 Alexa Fluor 594 (Invitrogen). The $\mathrm{pH}$ was adjusted to 7.4 with $\mathrm{CsOH}$. The reversal potential for chloride $\left(\mathrm{E}_{\mathrm{Cl}}\right)$ was calculated to be $-59.1 \mathrm{mV}$. Patch-clamp recordings were made with a 
MultiClamp 700B amplifier (Molecular Devices). Analog signals were filtered at $1 \mathrm{kHz}$ and sampled at $2 \mathrm{kHz}$ with the Digita Data $1400 \mathrm{~A} / \mathrm{D}$ board (Molecular Devices).

Analysis of physiological data. Except for the voltage-clamp data presented, each $n$ represents the average of 2-4 trials. Data are presented as mean \pm SEM. To identify statistical differences, $t$ tests were used when the data had a normal distribution. When the data did not possess a normal distribution, differences between two groups were tested using the Mann-Whitney rank sum test.

\section{Colocalization of directionally selective ganglion cells and $\mathrm{GFP}^{+}$} bipolar cells

Electrophysiologically identified DSGCs were dialyzed with Alexa Fluor 594 and visualized at $850 \mathrm{~nm}$ with a two-photon scanning laser. GFP ${ }^{+}$ bipolar cells were also imaged at $850 \mathrm{~nm}$ so that red and green channels could be captured consecutively, allowing for direct comparison of Z-projections. Z-stack reconstructions and pseudo-color merging were performed using ImageJ software (http://rsbweb.nih.gov/ij/). The Z-resolution of our two-photon system was $2.4 \mu \mathrm{m}$ (full width at half max fluorescence). Contact points between DSGC ON dendrites and Type 7 bipolar cell terminals were assessed by selecting a $50 \times 50 \mu \mathrm{m}$ area and identifying contacts points in the Z-plane. To test for significance of contacts, we rotated the ganglion cell channel $180^{\circ}$ out of phase and compared the number of contacts point to control.

\section{Results}

\section{Vsx1 is expressed in Type 7 ON bipolar cells}

In addition to its expression and requirement in OFF cone bipolar cells, the homeodomain transcription factor Vsx1 is also expressed in an uncharacterized subset of ON cone bipolar cells (Chow et al., 2004). To determine which of the five morphologically distinct ON cone bipolar cell types express $V s x 1$, we examined the morphology of ON bipolar cells expressing the $\tau$-LacZ knock-in reporter gene that has previously been targeted to the Vsxl locus (Chow et al., 2004). $\beta$-Galactosidase-immunolabeled retinal sections from adult mice were coimmunolabeled for the amacrine cell marker calretinin, as this marker labels three distinct bands in the inner plexiform layer that are used to classify bipolar cell types based on the stratification of their axon terminals (Ghosh et al., 2004). Vsx1: $\tau$ - $\beta$-galactosidaseexpressing $\mathrm{ON}$ cone bipolar cell axon terminals were detected just below the lowest calretinin band (i.e., closest to the ganglion cell layer) that corresponds to sublamina 4 of the inner plexiform layer (Fig. 1). The narrowly stratified axon terminals of these cells closely resemble Type 7 ON cone bipolar cells, which also project to sublamina 4 of the inner plexiform layer (Ghosh et al., 2004). Thus, by virtue of reporter labeling, $V s x 1$ appears to be expressed in Type 7 ON bipolar cells.

As no Type 7 bipolar cell-specific immunohistological markers have been identified, validation that the ON cone bipolar cells expressing the $V s x 1 \beta$-galactosidase reporter were Type 7 cells required an alternate approach. We therefore used the GUS8.4GFP transgenic reporter mouse line in which GFP reporter expression is directed by an $8.4 \mathrm{~kb}$ upstream promoter region of the $\alpha$-gustducin gene (Huang et al., 1999, 2003). GUS8.4GFP reporter expression strongly labels Type $7 \mathrm{ON}$ bipolar cells and is weakly expressed in rod bipolar cells (Huang et al., 2003). In wild-type mice carrying the GUS8.4GFP reporter, Vsx1 immunolabeling was detected in Type 7 ON bipolar cells expressing high levels of GFP (Fig. $2 A-C$, arrowheads) but not in rod bipolar cells expressing low levels of GFP (Fig. $2 A-C$, arrows). These observations are consistent with the previous finding that Vsx1 is not expressed in rod bipolar cells (Chow et al., 2001) and confirm the expression of $V s x 1$ in Type 7 bipolar cells.

We next crossed mice carrying the GUS8.4GFP transgenic reporter to mice harboring the $V s \times 1: \tau L a c Z$ knock-in reporter gene to determine whether the expression of GUS8.4GFP in Type 7
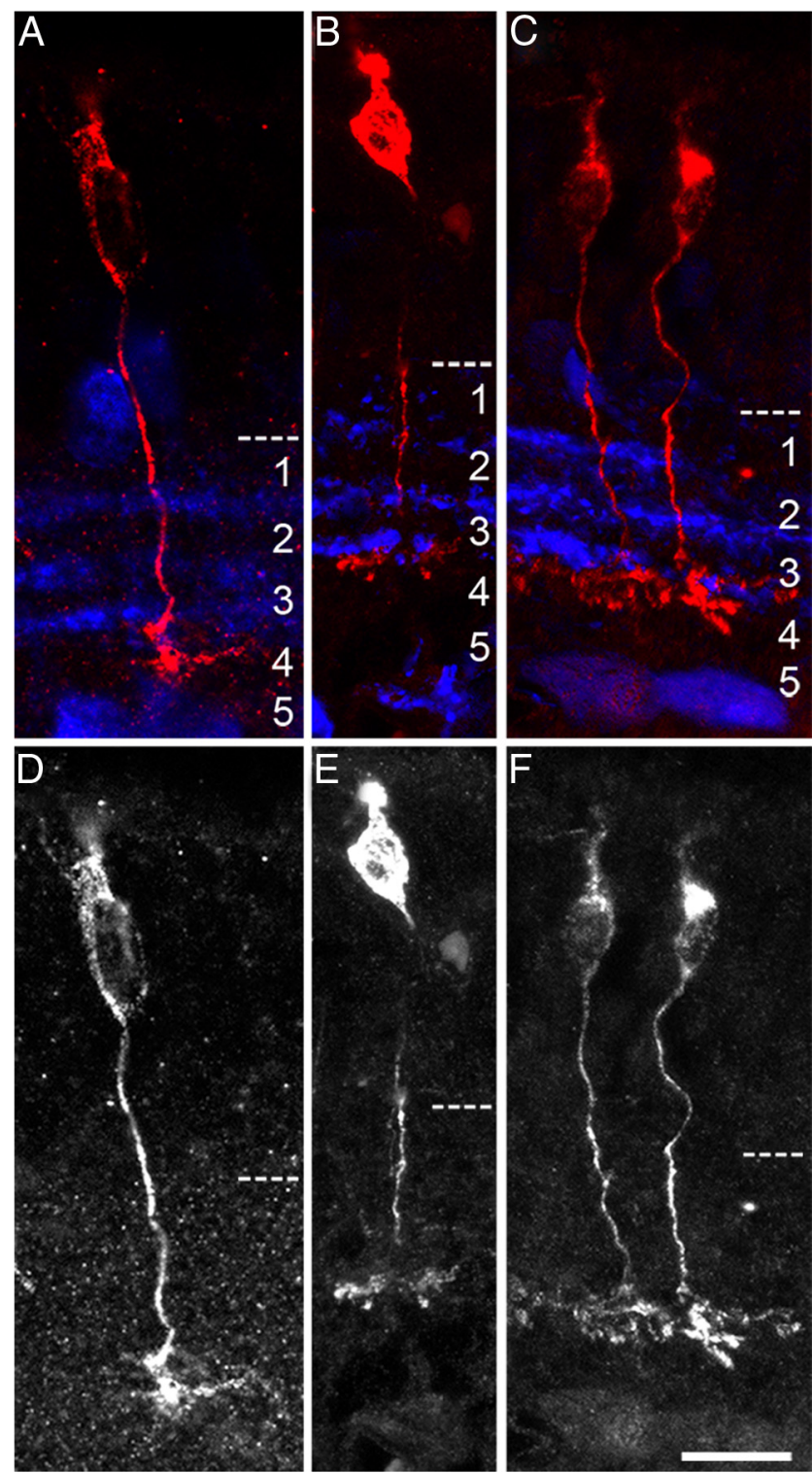

Figure 1. Morphological identification of Vsx1-expressing $0 \mathrm{~N}$ cone bipolar cell types in the Vs $\times 1^{\tau L a c Z /+}$ retina. $\beta$-Galactosidase immunolabeling of $0 \mathrm{~N}$ bipolar cells $(\boldsymbol{A}-\boldsymbol{C}$, red labeling; $\boldsymbol{D}-\boldsymbol{F}$, grayscale) in $V s \times 1^{\tau L a c Z /+}$ knock-in mice. Axon terminals of these cells are narrowly stratified and project to sublamina 4 of the inner plexiform layer, which is distinguished by calretinin immunolabeling in the inner plexiform layer ( $\boldsymbol{A}-\boldsymbol{C}$, blue labeling). Scale bar: (in $\boldsymbol{F}) \boldsymbol{A}-\boldsymbol{F}, 14 \mu \mathrm{m}$.

ON bipolar cells was affected by the loss of $V s x 1$ gene function. In

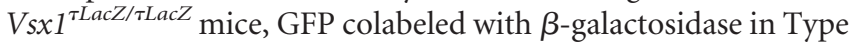
7 bipolar cells (Fig. 2 B). The axon terminals of GUS8.4GFP expressing Type 7 cells in $V s x 1^{\tau L a c Z / \tau L a c Z}$ mice were morphologically indistinguishable from those observed in wild-type mice (Fig. $3 A, B)$, indicating that $V s x 1$ is not required for the specification and gross morphogenesis of Type 7 bipolar cells. As the GUS8.4GFP reporter gene is functional in both wild-type and $V s \times 1^{\tau L a c Z / \tau L a c Z}$ mice, it therefore represents a useful genetic tool for examining the role of $V s x 1$ in Type $7 \mathrm{ON}$ bipolar cells.

\section{$V s x 1$ is required for the repression of gene expression in Type 7 bipolar cells}

Through the course of our studies using the GUS8.4GFP transgenic reporter, our observations suggested that the level of GFP

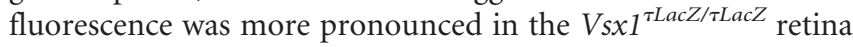




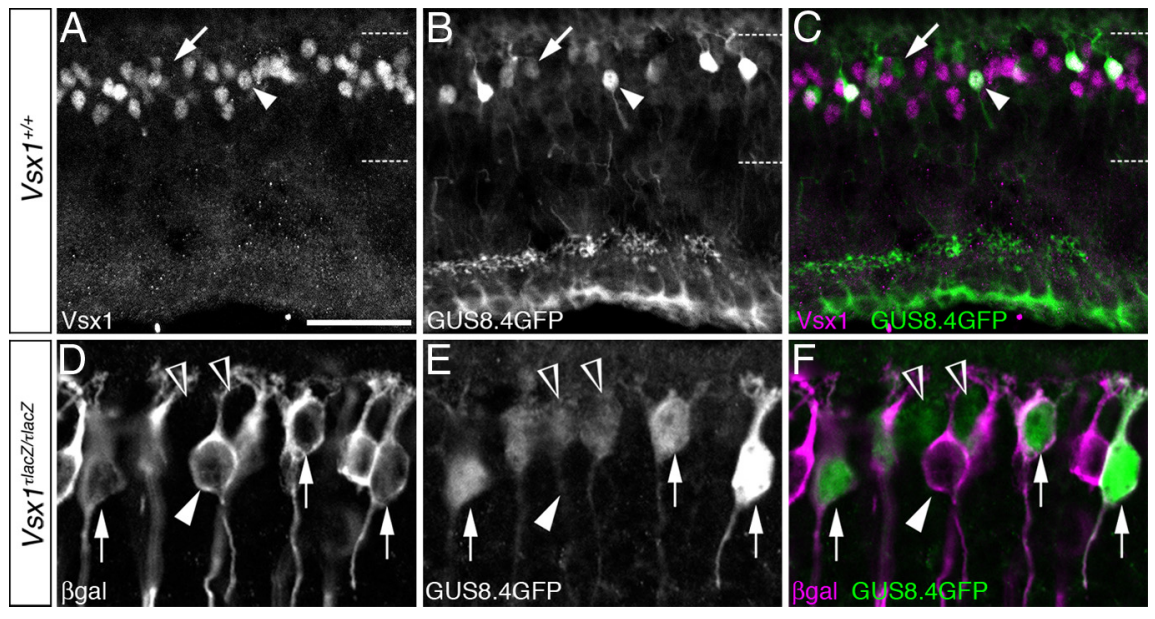

Figure 2. Colocalization of Vsx1 and the GUS8.4GFP reporter in putative Type $70 \mathrm{~N}$ cone bipolar cells. Vsx 1 immunolabeling $(\boldsymbol{A})$ in adult retinal sections from mice harboring the GUS8.4GFP reporter transgene is detected in putative Type 7 0N bipolar cells coimmunolabeled with GFP ( $\boldsymbol{B}$, arrowhead). The image in $\boldsymbol{C}$ shows the merged images of $\boldsymbol{A}$ and $\boldsymbol{B}$. Putative rod bipolar cells exhibiting low levels of GFP immunolabeling $\left(\boldsymbol{A}-\boldsymbol{C}\right.$, arrow) do not colabel with Vsx1. In Vsx $1^{\tau L a c Z / \tau L a c Z}$ mice, $\beta$-galactosidase (D) and GFP $(\boldsymbol{E})$ coimmunolabeling is observed in putative Type 7 bipolar cell ( $\boldsymbol{D}-\boldsymbol{F}$, arrows). Some $\beta$-galactosidase-expressing cells (D-F, white arrowhead) do not colabel with GFP, while some weakly immunolabeled GFP cells do not express the Vsx1: $\tau$ - $\beta$ galactosidase reporter ( $\boldsymbol{D}-\boldsymbol{F}$, open arrowheads). The dashed lines in $\boldsymbol{A}-\boldsymbol{C}$ indicate the boundary of the inner nuclear layer. Scale bars: $\boldsymbol{A}-\boldsymbol{C}, 30 \mu \mathrm{m} ; \boldsymbol{D}-\boldsymbol{F}, 15 \mu \mathrm{m}$.

than in the wild type (Fig. $3 A, B$ ). To examine the possibility that expression of the GUS8.4GFP reporter was regulated by $V s x 1$, Western blotting was used to compare the level of GFP in wild-

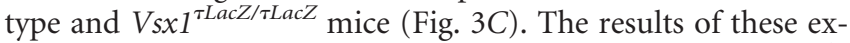
periments revealed an increase of GFP protein levels in $V s x 1^{\tau L a c Z /}$ ${ }_{\tau L a c Z}$ mice $(n=3)$ with up to a $75 \%$ increase in the total amount of GFP signal. These Western blot data, however, do not distinguish between increases in cell number and/or cell-intrinsic expression levels. We therefore used flow cytometry to quantify and compare the GUS8.4GFP-expressing cell populations in adult wild-type and $V s x 1^{\tau L a c Z / \tau L a c Z}$-dissociated retinas (Fig. 3D-G). Consistent with our Western blotting results, a $56 \%$ increase in mean GFP fluorescence intensity was observed for the GFP-

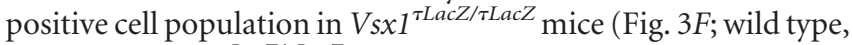
$411 \pm 39 ; V_{s} \times 1^{\text {LacZ/ } \tau \text { LacZ }}, 643 \pm 36$ arbitrary units measuring fluorescence intensity; $p<0.05$ ). In addition, while the overall proportion of cells expressing GFP was unchanged in $V_{s} \times 1^{\tau L a c Z /}$ ${ }_{\tau L a c Z}$ mice (Fig. $3 G$ ), there was a significant increase in the proportion of cells with high levels of GFP fluorescence (Fig. 3G; wild type, $\left.0.11 \pm 0.005 ; V s x 1^{\tau \text { LacZ/ } / \mathrm{Lac} Z}, 0.21 \pm 0.03, p<0.05\right)$. These

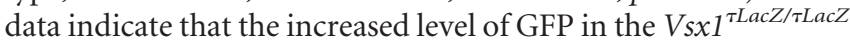
retina was due to elevated GFP expression per cell as opposed to an increase in the total number of cells expressing GFP. Combined, these data suggest that in the wild-type retina, $V s x 1$ partially represses GUS8.4GFP transgene expression in Type 7 bipolar cells.

We next undertook a quantitative approach to determine whether the $V s x 1$ homolog $C h x 10 / V s \times 2$ was specifically regulated in Type 7 bipolar cells. In the wild-type retina, Chx10 is expressed in all retinal bipolar cells (Burmeister et al., 1996); however, its expression level varies in different bipolar cells (Clark et al., 2008). Interestingly, the expression of Vsx1 and Chx10 in bipolar cells tends to be inversely related, suggesting that these transcription factors corepress each other (Clark et al., 2008). Consistent with this possibility, studies on the Chx10 ocular retardation J mutant have shown that $C h x 10$ negatively regulates $V s x 1$ expression (Clark et al., 2008). We were therefore interested in determining whether $V s \times 1$ might negatively regulate Chx10 expression. Chx10 immunofluorescence levels in Type 7 bipolar cells were com-

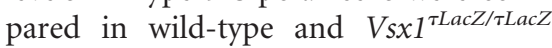
mice and normalized to Chx10 levels in rod bipolar cells colabeled with the rod bipolar cell-specific marker protein kinase $\mathrm{C} \alpha(\mathrm{PKC} \alpha$; Fig. $4 A-D, I)$. Normalizing Chx10 levels in this manner necessitated the assumption that Chx10 expression in rod bipolar cells is not affected by the loss of $V s x 1$. This assumption was based on the observation that $V s x 1$ is not expressed in rod bipolar cells, and no defects in rod bipolar gene expression or rod visual signaling defects are observed in $V s x 1^{\tau \text { LacZ/ }}$ ${ }^{\tau \text { LacZ }}$ mice (Chow et al., 2001, 2004). Using this approach, we observed a threefold upregulation of Chx10 in Type 7 bipolar cells in the Vsxl-null retina (0.202 \pm 0.018 in wild type compared to $0.610 \pm$ 0.018 in $V_{s} \times 1^{\tau \text { LacZ } / \tau \text { Lac } Z}$ mice, mean $\pm \mathrm{SE}$, $n=3$; Fig. 4I). We next examined

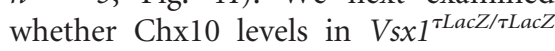
mice were altered in Type 1 or 2 OFF bipolar cells, as Vsxl is also expressed in these cells in wild-type mice. In contrast to Type 7 bipolar cells, the level of Chx10 immunofluorescence in NK3R-immunolabeled OFF bipolar cells did not change signifi-

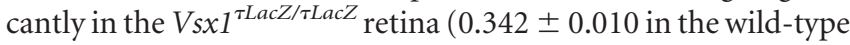
compared to $0.338 \pm 0.011$ in the $V s x 1$ null, mean $\pm \mathrm{SE}, n=3$; Fig. $4 E-I)$. These results reveal a cell type-specific regulatory role for $V s x 1$ in which it is required for the repression of Chx10 expression in Type 7 ON bipolar cells but not in Type 1 or 2 OFF bipolar cells.

As our findings suggested that $V s \times 1$ functions as a repressor of gene expression in Type 7 bipolar cells, we explored the possibility that other bipolar cell-specific markers might also be deregulated in the absence of $V s x 1$. Previous work has shown that in $V s \times 1^{\text {LacZ/ } / \text { LacZ }}$ retinal sections immunolabeled for the calciumbinding protein Cabp5 (a Type 3, 5, and rod bipolar-specific marker; Haverkamp et al., 2003), a characteristic "gap" in labeling that separates the axon terminals of Type 5 and rod bipolar cells is no longer evident (Chow et al., 2004). Normally, the "gap" between the axon terminals of Type 5 and rod bipolar cells coincides with the region of the inner plexiform layer where the axon terminals of Type 7 bipolar cells reside (Fig. 5G-I, arrows). Consistent with the hypothesis that Cabp5 is negatively regulated by $V s x 1$ in Type 7 bipolar cells, ectopic Cabp5 immunolabeling was

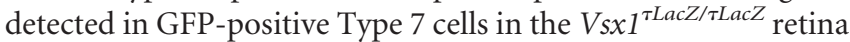
(Fig. 5, compare outlined cells in $E$ and $N$ and arrows in $G-J$ and $P-R)$. In contrast to Cabp5, the expression of other bipolar cell markers, including the Type $3 \mathrm{a}$ and $3 \mathrm{~b}$ cell markers hyperpolarization-activated and cyclic nucleotide-gated channel 4 (HCN4) and regulatory subunit RII- $\beta$ of protein kinase A (PKARII-beta; Mataruga et al., 2007), respectively, were not upregulated in Type 7 bipolar cells (data not shown). Together, these findings indicate that $V s \times 1$ is selectively required for the repression of gene expression in Type 7 bipolar cells.

GUS8.4GFP-expressing Type 7 bipolar cells cofasciculate with $\mathrm{ON}-\mathrm{OFF}$ directionally selective ganglion cells

Despite the initial finding of b-wave ERG deficits in $V s x 1^{\tau L a c Z / \tau L a c Z}$ mice, previous attempts at measuring bipolar cell output using 

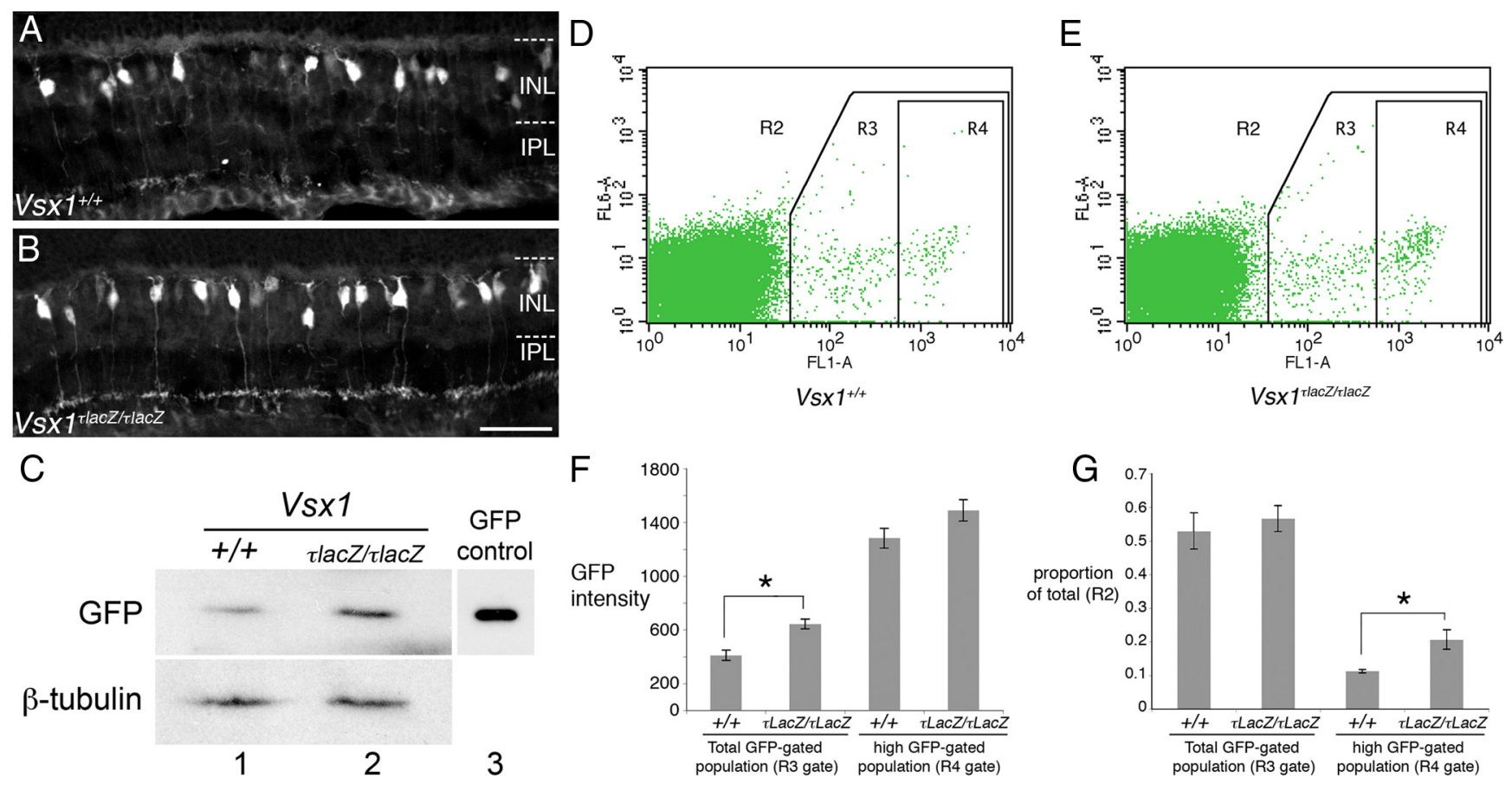

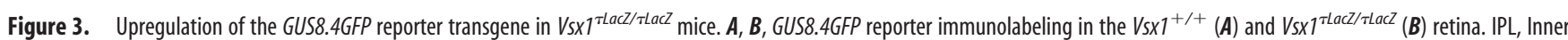
plexiform layer; INL, inner nuclear layer. C, Western blot showing GFP and control $\beta$-tubulin protein levels from total retinal lysates of Vsx ${ }^{+/+}$(column 1) and Vsx $1^{\tau L a c Z / \tau L a c Z}$ (column 2) mice. 293 cells transfected with a GFP-expressing plasmid were used as a positive control for GFP detection (column 3). D-G, Flow cytometry data were obtained from papain-dissociated retinas from $V_{s \times 1} 1^{+/+}$and $V_{s x} 1^{\tau L a C Z / \tau L a C Z}$ mice carrying the GUS8.4GFP transgene. Representative examples showing the forward and side scatter of cell populations designated as R2 (i.e., all of the cells within the plot) are shown for $V s x 1^{+/+}(\boldsymbol{D})$ and $V s x 1^{\tau L a c Z / \tau L a c Z}(\boldsymbol{E})$ mice. $\boldsymbol{E}$, Relative mean fluorescence in the GFP-positive population indicated by R3 (i.e., all of the cells within the R3 box) was significantly

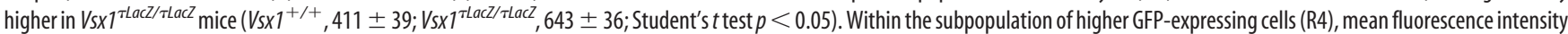
was not significantly increased $(\boldsymbol{F})$, indicating that highly fluorescing cells in $V s \times 1^{+/+}$mice were not getting brighter in Vsx $1^{\tau L a c z / \tau L a c z}$ mice. Although the total number of GFP fluorescing cells (R3/R2) was unchanged in Vsx $7^{\text {tlacz/tlacz }}$ mice $(\boldsymbol{G})$, a significant increase in the number of cells with high GFP fluorescence (R4/R2) was observed $\left(\boldsymbol{F}, \boldsymbol{G}^{*}{ }^{*} p<0.05\right.$ by Student's t test). Scale bar: (in B) $A, B, 35 \mu \mathrm{m}$.

electrophysiological single-unit recordings from ON and OFF ganglion cells revealed defects in only the OFF system (Chow et al., 2004). It is possible that no defect in the ON system was found due to the fact that $V s x 1$ is only expressed in a small subset of the six known ON cone bipolar cell types in the mouse retina (Wässle et al., 2009). To more specifically test for defects in the ON system, we recorded from ON-OFF directionally selective ganglion cells, DSGCs, which have putatively been shown to receive direct input from Type 7 ON bipolar cells (Lin and Masland, 2005). DSGCs were identified by their medium-sized elliptical somas, their bistratified dendritic arbors, and their directional preferences to moving light stimuli. Labeled ON-OFF DSGCs showed apparent cofasciculation with Type 7 bipolar cells in both wildtype and $V_{s x} 1^{\text {LLacZ } / \tau L a c Z}$ retinas (Fig. $6 ; n=3$ for wild type; $n=3$

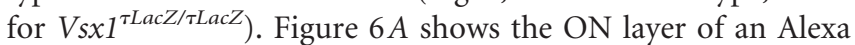

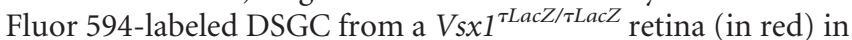
relation to Type 7 bipolar cell terminals (in green). Figure $6, B-D$, shows two higher-magnification views of DSGC dendrites (Fig. $6 B$ ), Type 7 bipolar cell terminals (Fig. 6C), and a merge of both (Fig. 6D). Cofasciculation between DSGC dendrites and Type 7 bipolar cell terminals are shown in Figure $6 D$ as yellow. Figure $6 E$ shows a Z-projection of Figure $6 \mathrm{D}$ rotated $90^{\circ}$. Cofasciculation was verified by colocalization (in yellow) of DSGC dendrites and Type 7 bipolar cell terminals in the Z-projection (Fig. 6E). As cofasciculation at the level of light microscopy is insufficient evidence of synaptic connections, we next rotated the ganglion cell channel $180^{\circ}$ out of phase and assessed whether this affected the apparent colocalization. Rotation of one channel resulted in significantly less apparent colocalization $(14.1 \pm 2.5$ contacts/2500 $\mu \mathrm{m}^{2}$ for control compared to $8.1 \pm 1.2$ contacts $/ 2500 \mu \mathrm{m}^{2}$ after $180^{\circ}$ rotation, suggesting that approximately half of the apparent contact points were putative synapses). Based on the estimated dendritic area of the ON subfield of DSGCs $\left(\sim 40,000 \mu \mathrm{m}^{2} / 2\right.$; Coombs et al., 2006) there appear to be $\sim 112$ Type 7 ON bipolar synapses onto a given DSGC. Since a single DSGC has $\sim 667$ ON synapses (synaptic density of $\sim 1$ synapse/3 $\mu \mathrm{m}$ on $\sim 2000 \mu \mathrm{m}$ of ON dendrites; Coombs et al., 2006; Koizumi et al., 2011), the contacts made by Type 7 ON bipolar cells likely represent only about $1 / 5$ of the total number of synapses in the ON subfield. Together, these data indicate that ON-OFF DSGCs likely receive input from multiple types of ON bipolar cells, including Type 7

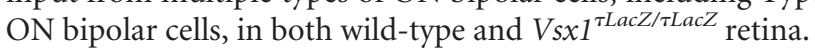

\section{ON responses of ON-OFF DSGCs are more sustained in $V s x 1^{\tau L a c Z / \tau L a c Z}$ mice}

To assess possible defects in the ON signaling pathway in Vsx $1^{\text {LLacZ/rLacZ }}$ mice, we first examined bipolar cell output by recording stationary flash responses from ON-OFF DSGCs in wild-

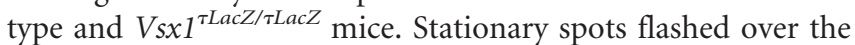
cell elicited robust $\mathrm{ON}$ and OFF responses in both wild-type and $V_{s x} 1^{\text {TLacZ/rLacZ }}$ ON-OFF DSGCs (Fig. $7 A, B$; the spot size was optimized for each cell to elicit a maximal response). When spots were presented, ON responses in wild-type and $V s x 1^{\text {tLacZ/rLacZ }}$ DSGCs had similar peak spike rates (Fig. 7C; $p>0.05$ ) as well as response onset latencies (data not shown; $p>0.05$ ), but the total number of ON spikes evoked by a $2 \mathrm{~s}$ flash was greater in the $V_{s x} 1^{\text {TLacZ/rLacZ }}$ mice (Fig. $7 D ; 18.5 \pm 3.9$ for wild type compared to $37.9 \pm 6.7$ for knock-out; $p<0.05 ; n=8$ for wild type; $n=10$ 


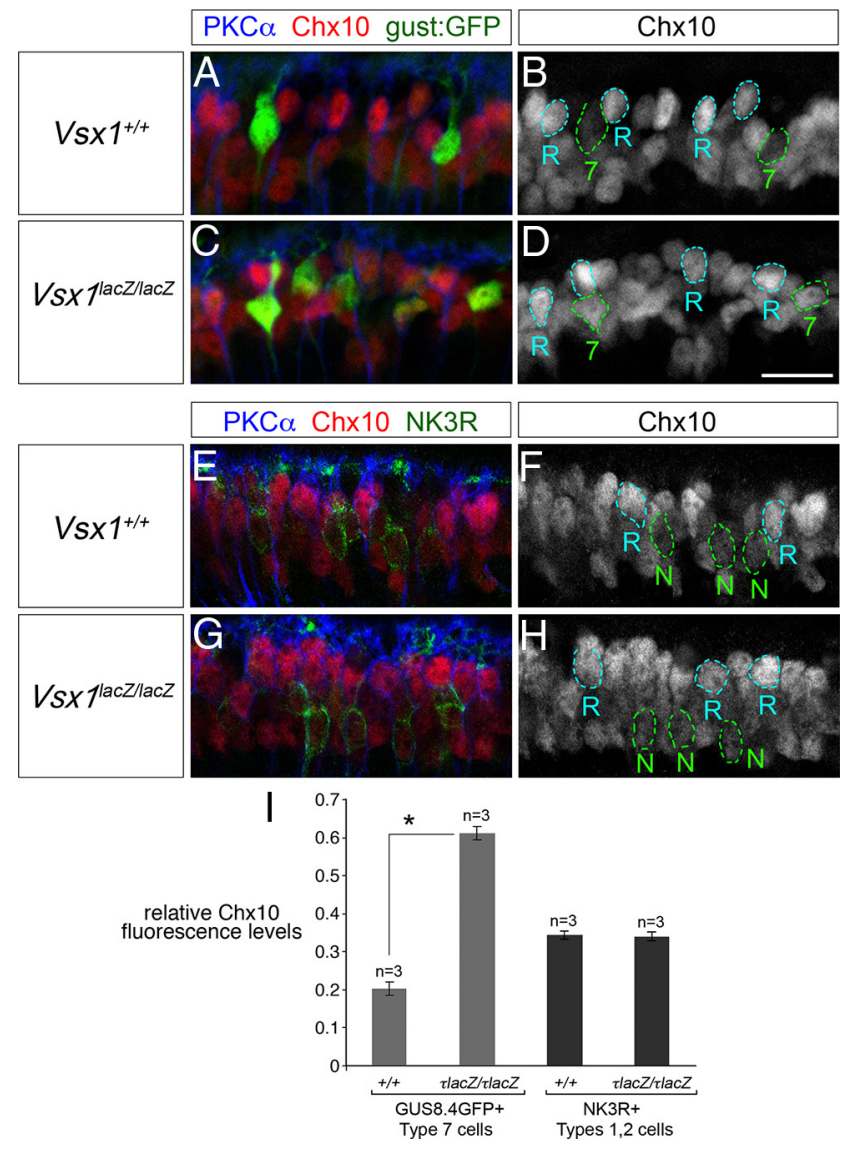

Figure 4. Upregulation of Chx10 in Type 7 bipolar cells in Vsx $7^{\text {TLacZ/ } / L a c Z}$ mice. $\boldsymbol{A}-\boldsymbol{H}$, Immunolabeling of adult retinal sections for Chx10, PKC $\alpha$, and the GUS8.4GFP (gust:GFP) reporter $(\boldsymbol{A}-\boldsymbol{D})$ and $\mathrm{Chx} 10$, PKC $\alpha$, and NK3R $(\boldsymbol{E}-\boldsymbol{H})$. Chx10 immunofluorescence in Type 7 bipolar cells is outlined in green and labeled " 7 " in $\boldsymbol{B}$ and $\boldsymbol{D}$. Chx10 immunofluorescence in PKC $\alpha$-positive rod bipolar cells is outlined in blue and labeled " $\mathrm{R}$ " in $\boldsymbol{B}, \boldsymbol{D}, \boldsymbol{F}$, and $\boldsymbol{H}$. NK3R-immunolabeled Type 1 and $20 \mathrm{FF}$ bipolar cells are outlined in green lines and labeled " $\mathrm{N}$ " in $\boldsymbol{F}$ and $\boldsymbol{H}$. I, Chx10 immunofluorescence levels in Type 7 or Type $1 / 2$ bipolar cells was normalized to Chx10 immunofluorescence levels in rod bipolar cells and compared in $V_{s x} 1^{+/+}$and $V s \times 1^{\text {tlacZ/ } / \text { LacZ }}$ mice. In the Vsx $7^{\text {TLacZ//LacZ }}$ retina, Chx10 immunofluorescence is upregulated in Type 7 bipolar cells to almost three times of that observed in the wild-type retina $\left(0.610 \pm 0.018\right.$ in Vsx $1^{\text {TlacZ/ } / \text { LacZ }}$ compared to $0.202 \pm 0.018$ in $V s \times 1^{+/+}$, mean $\pm S E, n=3$ ). The level of Chx10 immunofluorescence does not change significantly in NK3R immunolabeled OFF bipolar cells in the

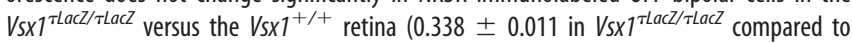
$0.342 \pm 0.010$ in $V_{s \times} 1^{+/+}$, mean $\pm S E, n=3$ ). The asterisk in $I$ indicates a significant difference by Student's $t$ test $\left({ }^{*} p<0.01\right)$. Scale bar: (in $\left.\boldsymbol{D}\right) \boldsymbol{A}-\boldsymbol{H}, 14 \mu \mathrm{m}$.

for knock-out). In contrast, no differences were observed in the OFF responses in DSGCs (unlike previous findings for OFF non-DS ganglion cell responses; see below). Since the peak spike rate of the ON response in $V s x 1^{\tau L a c Z / \tau L a c Z}$ did not differ from that in wild type, but the total spike number increased, it suggested that the ON response was more sustained. Indeed, the time from the first to the last spike generated after the onset of the light

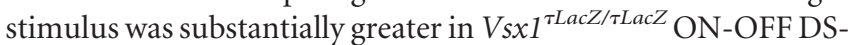
GCs (931 $\pm 162 \mathrm{~ms} ; n=8)$ as compared to wild type (289 \pm 44 ms; $n=10 ; p<0.05$; Fig. $7 E)$. It is interesting to note that we did not find deficits in OFF signaling as has been seen previously (Chow et al., 2004). However, in this previous study, ON-OFF ganglion cells were excluded from the analysis. Furthermore, it remains unclear whether ON-OFF DSGCs receive input from $V s x 1$-expressing OFF bipolar cells. Finally, the OFF deficits appeared mainly at bright light levels (Chow et al., 2004). Therefore, further experiments using increasing light intensities would be needed to test whether ON-OFF DSGCs show OFF signaling impairments similar to what has been found previously for Vs $x 1$ null mice. Together, our results indicate a selective impairment of the ON response of ON-OFF DSGCs in $V s x 1^{\tau L a c Z / \tau L a c Z}$ retina.

To assess whether $V s x 1$ inactivation affected specific circuits or the whole ON network, we compared responses from other types of ON ganglion cells in $V s x 1^{\tau L a c Z / \tau L a c Z}$ and wild-type retina. Spiking responses of ON cells were compared using a transientness index, which was defined as the number of spikes within the first $200 \mathrm{~ms}$ of light stimulation divided by the total number of ON spikes (values between 0 and 1, with larger values indicating more transientness). We found no difference in the transientness

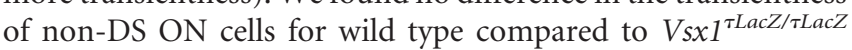
(Fig. 7F; $0.30 \pm 0.08$ for wild type compared to $0.29 \pm 0.07$ for $\mathrm{KO} ; p>0.05 ; n=9$ for wt; $n=13$ for $\left.V s x 1^{\tau \text { LacZ } / \tau L a c Z}\right)$, although with this analysis we did find a significant difference between ON

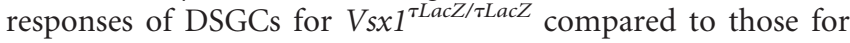
wild type $(0.75 \pm 0.05$ for wild type compared to $0.58 \pm 0.04$ for $\left.V s \times 1^{\text {rLacZ/ } / \text { LacZ }} ; p<0.05\right)$. These results show that $\mathrm{ON}$ response defects appear to be limited to ON-OFF DSGCs. However, it is also possible that other $\mathrm{ON}$ circuits are affected, but when probed using a blind approach combining multiple ON circuits, these are not apparent (Chow et al., 2004). Future studies will reveal whether other specific types of ON ganglion cells are affected in Vsx1-null mice.

To ensure that the more sustained $\mathrm{ON}$ responses of $V s x 1^{\tau L a c Z / \tau L a c Z}$ DSGCs were a result of increased excitatory input to ON-OFF DSGCs and not from changes in circuit properties or from postsynaptic changes within the ganglion cells themselves, we next measured EPSCs from these cells. EPSCs from $V s x 1^{\tau L a c Z / \tau L a c Z}$ retina, measured at $-60 \mathrm{mV}$ to isolate excitatory currents, had more sustained $\mathrm{ON}$ responses compared to control, consistent with spike recording data. Figure $8, A$ and $B$, show representative traces of $O N$ and OFF excitatory current responses, respectively, normalized to

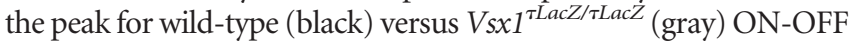
DSGCs. Comparison of the peak currents for wild-type and

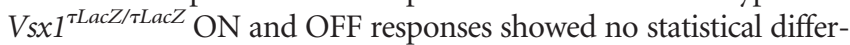
ence $(p>0.5$; Fig. $8 C)$, consistent with the finding that the peak spike rate did not change under the same conditions (Fig. 7C). To compare the kinetics of the ON and OFF EPSCs, the rise and decay slopes were estimated with a single exponential function. The decay time constant $\left(\tau_{\mathrm{d}}\right)$ of the ON response for Vsx1-null DSGCs was found to be much larger compared to control (Fig. 8D; $340 \pm 72 \mathrm{~ms}$ for

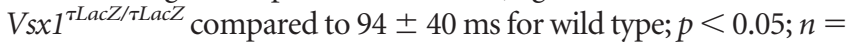

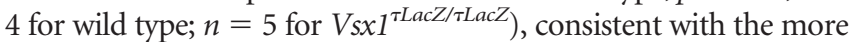
sustained spiking $\mathrm{ON}$ responses observed in previous experiments. No difference was seen in decay or rise times for OFF responses

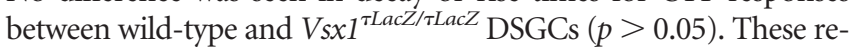

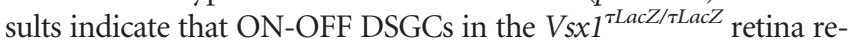
ceive more sustained excitatory input during the $\mathrm{ON}$ response compared to wild type, whereas the OFF response appears unchanged.

Directional selectivity is decreased in $V s x 1^{\tau L a c Z / \tau L a c Z}$ mice As directionally selective responses are known to be generated by a finely controlled interaction between excitatory and inhibitory pathways (Taylor and Vaney, 2002), it was conceivable that the altered kinetics of the ON response in Vsx1-null DSGCs might affect their ability to compute direction. To test this possibility, we next compared responses to moving stimuli in $V s x 1$-null and wild-type DSGCs. Robust responses were evoked by the leading $(\mathrm{ON})$ and trailing (OFF) edges of moving spots (400 $\mu \mathrm{m}$ diameter moving at $1000 \mu \mathrm{m} / \mathrm{s}$ ). Figure 9, $A$ and $B$, compares the re- 
sponses measured in wild-type and Vsx1null DSGCs, respectively. Polar plots, in which the average number of spikes over a number of trials $(2-4)$ is plotted against the direction of light stimulus, indicate that direction selectivity was maintained in Vsx1-null ganglion cells. However, the DSI (which ranges between 1 and 0 with larger values indicating stronger directional selectivity; see Materials and Methods) was significantly decreased for Vsx1null DSGCs compared to wild-type DSGCs for ON but not OFF spike responses (Fig. 9C; ON DSI was $0.72 \pm 0.07$ for wild type compared to $0.34 \pm 0.08$ for $V$ sx1-null DSGCs; $p<0.05 ; n=4$ for both wild type and knock-out). To ensure that presynaptic inhibitory mechanisms known to generate directional selectivity were still present in Vsx1-null mice, we measured IPSCs in DSGCs. In all ON-OFF DSGCs tested, IPSCs (measured at $0 \mathrm{mV}$ ) were larger for null direction stimuli as compared to preferred direction stimuli (data not shown; $n=3$ for wild type; $n=6$ for $V$ sx1-null mice; $p<0.05)$. This suggests that the usual inhibitory mechanisms known to generate DS (Fried et al., 2002) are intact in Vsx1-null mice. However, these inhibitory inputs are not as effective in blocking nulldirection spiking, thereby resulting in a reduced directional selectivity in the Vsx1-null retina.

\section{Discussion}

We used molecular, genetic, and electrophysiological approaches to examine the role of the paired-like homeodomain transcription factor $V s x 1$ in Type 7 ON bipolar cell terminal differentiation. We found that $V s x 1$ is required for the negative regulation of gene expression in Type 7 bipolar cells. This contrasts the role of $V s x 1$ in OFF bipolar cells where it is required for the positive regulation of gene expression. We also show that $V s \times 1$ is necessary for proper ON pathway visual signaling within a defined, directionally selective, retinal circuit.

\section{Vsx1 has differential effects in $\mathrm{ON}$ versus OFF bipolar cells}

Our study reveals a trend in which $V s x 1$ appears to be required for activation of gene expression in OFF bipolar cells and for repression of gene expression in Type 7 bipolar cells (Fig. 10). Specifically, in Vsx1-null mice Cabp5, Chx10, and GUS8.4GFP are upregulated in Type 7 ON bipolar cells, whereas the expression of seven OFF bipolar cell type markers, including recoverin, Neto1, and Syt2 (Type 2), NK3R (Types 1 and 2), Cabp5 (Type 3) (Chow et al., 2004; Ohtoshi et al., 2004), and HCN4 (Type 3a) (Shi et al., 2011) is downregulated. Interestingly, although it is not known whether the $\alpha$-gustducin gene is normally expressed in Type 7 bipolar cells, an analysis of the

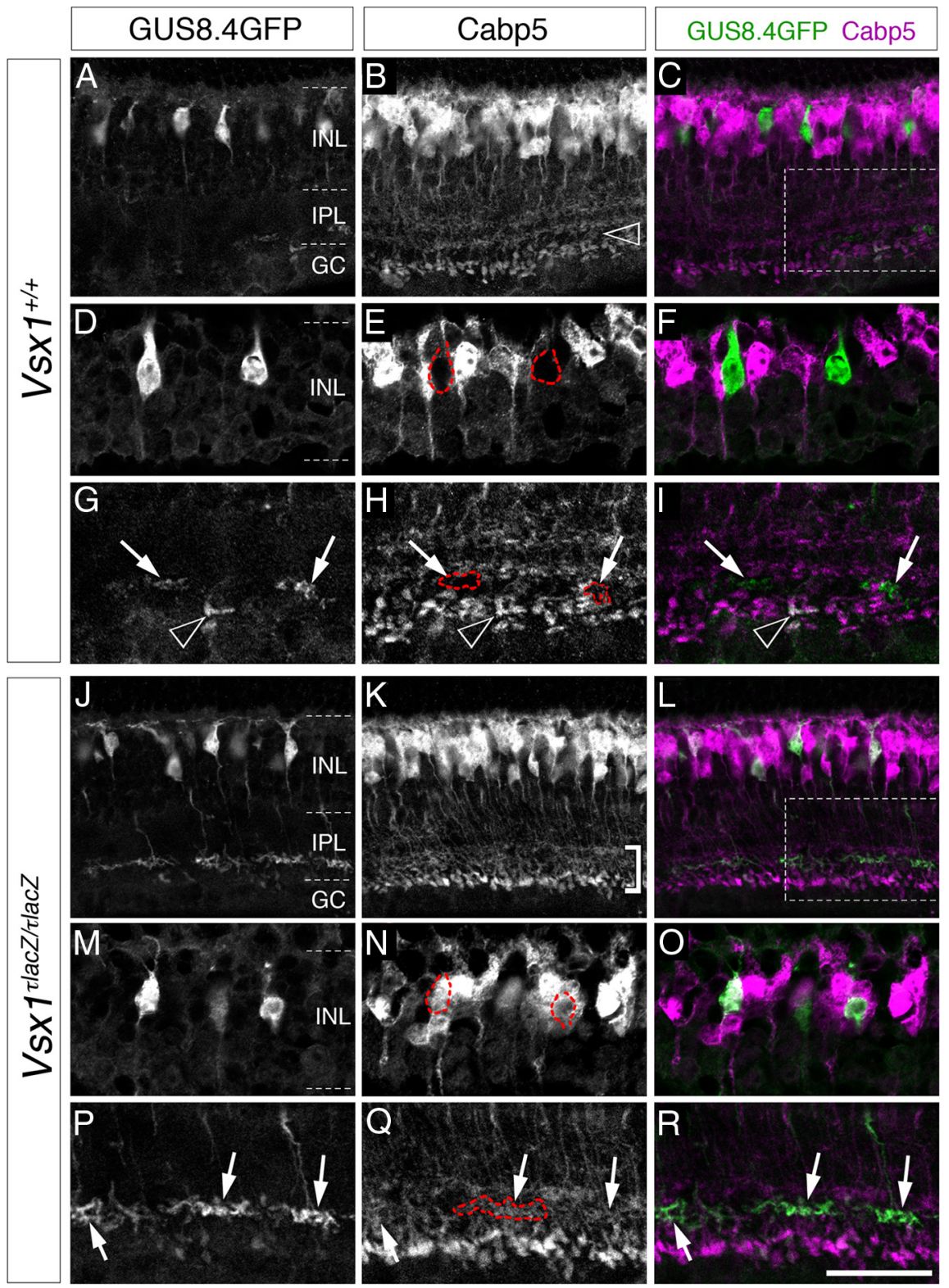

Figure 5. Cabp5 is ectopically expressed in Type 7 bipolar cells in $V s x 1^{\text {TLacZ/ } \tau L a c Z}$ mice. In the $V s x 1^{+/+}$retina, Calcium-binding protein 5, Cabp5, is expressed in a subset of bipolar cells (Type $30 \mathrm{FF}$ and Type $50 \mathrm{~N}$ and rod bipolar cells) (Haverkamp et al., 2003) and is normally not detected in putative Type 7 bipolar cells distinguished by their high level of fluorescence $(\boldsymbol{A}-\boldsymbol{I})$. The absence of Cabp5 in Type 7 cells is highlighted by red dashed for GFP-expressing soma $(\boldsymbol{E})$ and axon terminals ( $\mathbf{G}-\boldsymbol{I}$, arrows). The black arrowhead in $\mathbf{G}-\boldsymbol{I}$ points to the axon terminal of a GFP-expressing rod bipolar cell (distinguished by its position and shape) that is colabeled with Cabp5. In the $V_{s \times 1} 1^{+/+}$retina, a characteristic gap in Cabp5 immunolabeling is observed between the axon

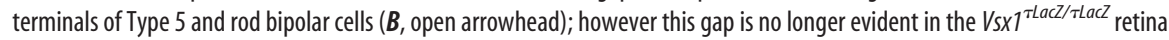
[bracketed region in K (Chow et al., 2004)]. Within this region of the inner plexiform layer, Cabp5 immunolabeling colabels with GFP in the axon terminals of Type 7 bipolar cells ( $\boldsymbol{J}-\boldsymbol{L}$; arrows in $\boldsymbol{P}-\boldsymbol{R}$ and red-outlined axon terminals in $\mathbf{Q}$ ). Colocalization of Cabp5 immunolabeling with the GUS8.4GFP reporter in the $V_{s x} 7^{\text {TLaCZ/ } \tau \text { LacZ }}$ retina was also observed in the cell bodies of putative Type 7 cells $(\boldsymbol{M}-\mathbf{0}$, outlined in red in $\boldsymbol{N})$. The boxed regions in ( and $\mathrm{L}$ are shown at higher magnification in $(\mathbf{G}-\boldsymbol{I})$ and $(\boldsymbol{P}-\boldsymbol{R})$, respectively. IPL, Inner plexiform layer; INL, inner nuclear layer; $G C$, ganglion cell layer. Scale bar: (in $\boldsymbol{R}) \boldsymbol{A}-\boldsymbol{C}, \boldsymbol{J}, \boldsymbol{K}, 40 \mu \mathrm{m} ; \boldsymbol{D}-\mathbf{G}, \boldsymbol{M}-\boldsymbol{R}, 20 \mu \mathrm{m}$.

$8.4 \mathrm{~kb}$ upstream region of the $\alpha$-gustducin gene used in the $\alpha$-gustducin reporter transgene contains at least seven predicted Vsx1 DNA binding sites (Z. Shi and R. L. Chow, data not shown). Together, these results suggest that Vsx1 is directly involved in transcriptional repression in Type 7 bipolar cells, although at this point further work is required to determine whether this is mediated through direct or indirect mechanisms. The different functions of Vsx1 in ON and OFF bipolar cells are therefore likely to involve cell type-specific differences in transcription factor 
A

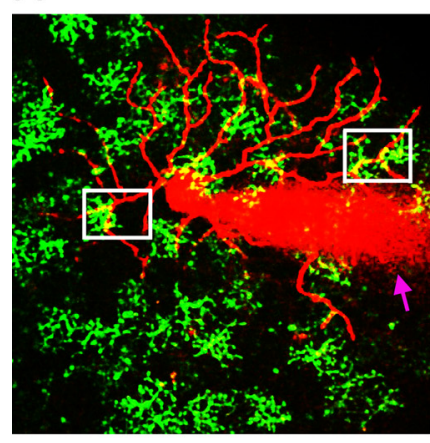

$50 \mu \mathrm{m}$
B
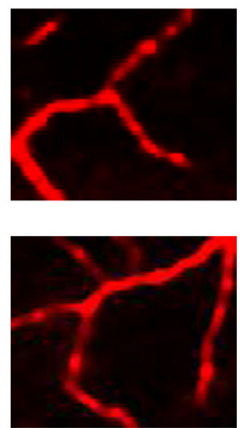

$\overline{10 \mu \mathrm{m}}$
C
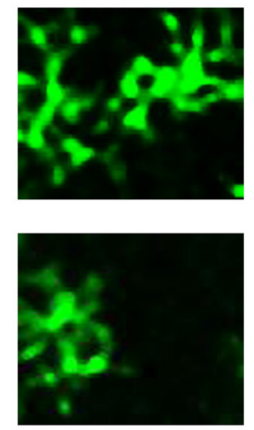

D
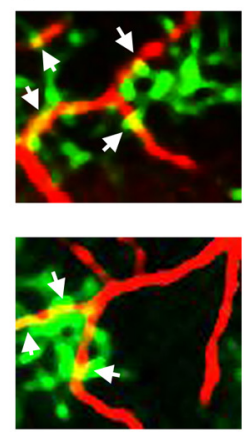

E
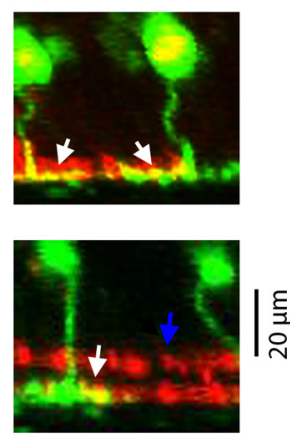

Figure 6. ON-OFF directionally selective ganglion cells appear to cofasciculate with Type $70 \mathrm{~N}$ bipolar cells. ON-OFF directionally selective ganglion cells were dialyzed with Alexa Fluor 594 and appear in the figure as red. GUS8.4GFP-expressing Type 7 bipolar cells appear as green. A, ON-OFF DSGC in relation to the axon terminals of Type 7 ON bipolar cells in a wild-type retina. Magnified versions of the areas in the white squares are shown in $\boldsymbol{B}-\boldsymbol{E}$. $\boldsymbol{B}$, ON dendrites from the labeled ON-OFF DSGC. $\boldsymbol{C}$, The axon terminals for Type 7 bipolar cells in the same areas. $\boldsymbol{D}, A$ merge of $\boldsymbol{B}$ and $\boldsymbol{C}$ showing apparent colocalization in yellow (pointed to by white arrows) of ON-OFF DSGC dendrites and Type 7 bipolar cell terminals. $\boldsymbol{E}$, Projection of the same area. The white arrow in $\boldsymbol{E}$ shows apparent colocalization between $0 \mathrm{~N}-0 \mathrm{FF}$ DSGC dendrites and Type 7 bipolar cell terminals. The blue arrow in $\boldsymbol{E}$ points to the $0 \mathrm{FF}$ dendritic layer. The magenta arrow in $A$ points to the recording electrode. Apparent colocalization was seen between Type 7 bipolar cell terminals and ON-OFF DSGC ON dendrites for all cells tested $(n=3$ for wild type; $n=3$ for control).

A

wt

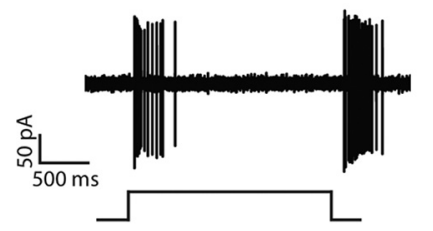

C

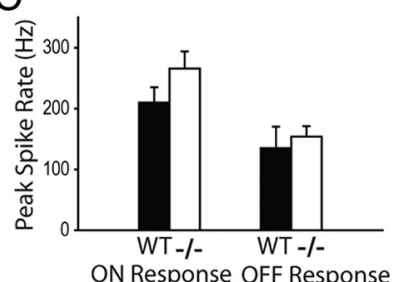

$\mathrm{E}$

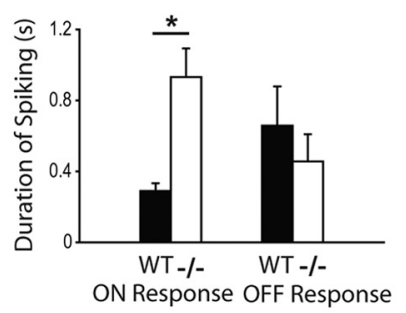

A

$-60 \mathrm{mV}$

B
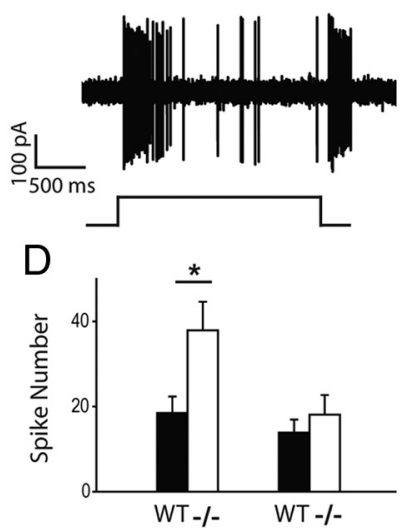

ON Response OFF Response

F

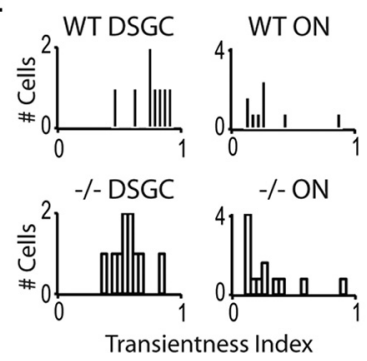

Figure 7. The $0 \mathrm{~N}$ spiking response of $V_{s \times} 7^{\tau L a c Z / \tau L a c Z} \mathrm{ON}-0 \mathrm{FF}$ directionally selective ganglion cells is more sustained. $A, B$, Representative spiking data of the flash responses from

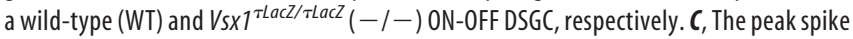

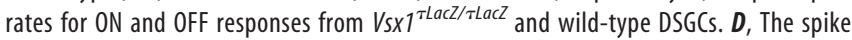
number for $0 \mathrm{~N}$ and $0 \mathrm{FF}$ responses from $V s \times 1^{\tau L a c Z / \tau L a c Z}$ and wild-type DSGCS. E, Duration of

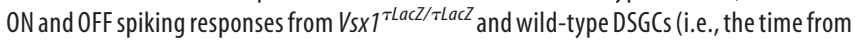
the first spike to the last spike generated by either the $\mathrm{ON}$ or OFF flash). $\boldsymbol{F}$, The transientness of ON spiking responses from WT and Vsx1-null DSGCs and non-DSGCs. Responses are plotted on a transientness index, calculated as the number for spikes elicited in the first $200 \mathrm{~ms}$ of the $\mathrm{ON}$ response divided over the total number of $\mathrm{ON}$ spikes. Values range from 0 to 1 , with higher values indicating more transient responses. For $\boldsymbol{C}-\boldsymbol{E}, n=8$ for wt; $n=$ 10 for knock-out. For $\boldsymbol{F}, n=9$ for wt; $n=13$ for knockout; ${ }^{*} p<0.05$.

A ON
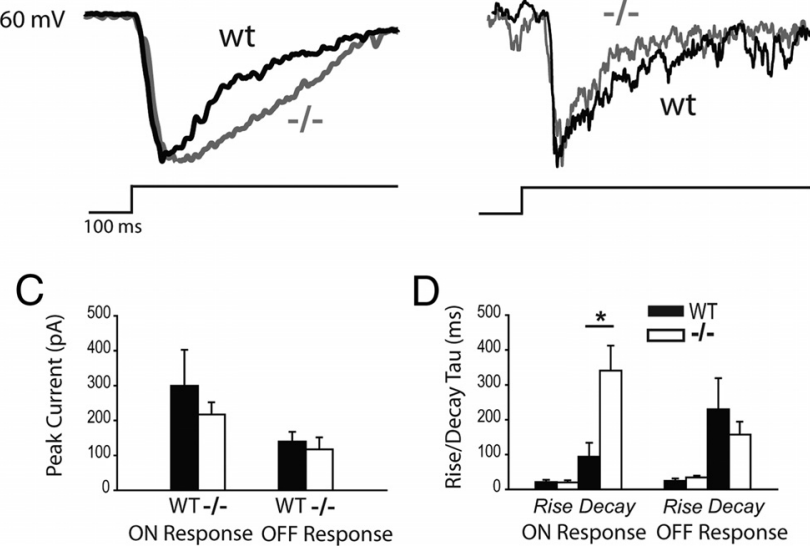

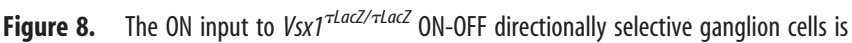
more sustained. $A, B$, Representative voltage-clamp data of the flash responses from a wild-

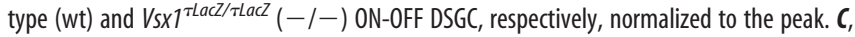
Plots of the peak ON and OFF currents for DSGCs from Vsx1-null and wild-type (WT) retina elicited by flashes. $D$, Plots the rise and decay time constants of ON and OFF EPSCs; $n=5$ for Vs $\times 7^{{ }^{\text {LLaCZ/ } / \text { LacZ }}} ; n=4$ for wild type; ${ }^{*} p<0.05$.

composition. Previous work has shown that the homeodomain transcription factor Irx5 and the bHLH transcription factor Bhlhb5 are coexpressed with Vsx1 in OFF bipolar cells and have overlapping and nonoverlapping loss-of-function phenotypes compared to $V s x 1$ that also lead to the downregulation of Types 2 and 3 bipolar cell markers (Cheng et al., 2005; Feng et al., 2006). Although these transcription factors may function in a combinatorial manner to activate gene expression in differentiating OFF bipolar cells, it is interesting to note that Vsx1, Irx5, and Bhlhb5 have all been characterized as transcriptional repressors in vitro (Xu et al., 2002; Bilioni et al., 2005; Dorval et al., 2005). Since homeodomain proteins can act as both transcriptional repressors and activators through their interaction with transcriptional cofactors (Wolberger, 1999; Reményi et al., 2004; Svingen and Tonissen, 2006), understanding how Irx 5 and Bhlhb5 as well as other transcription factors interact with Vsx1 to regulate the differential gene expression in Type 7 and OFF bipolar cells repre- 
sents an important next step in understanding the regulatory mechanisms that underlie retinal bipolar cell type diversity.

Interestingly, one of the targets repressed by $V s x 1$ in Type $7 \mathrm{ON}$ bipolar cells is its homolog, Chx10. Previous studies have shown that Chx10 is able to negatively regulate the expression of Vsx1 by directly binding to the $V s \times 1$ promoter region (Clark et al., 2008). Together, these findings suggest that Vsx1 and Chx10 cross-repress each other's expression and are consistent with in vitro experiments showing that both of these transcription factors can function as repressors (Dorval et al., 2005) and tend to exhibit inverse expression patterns in bipolar cells (Clark et al., 2008). Since the expression of Chx10 in Type 2 bipolar cells is not dependent on Vsx1 (this study), our data further indicate that Vsx1-Chx10 crossrepression is complex and likely involves the participation of other cell type-specific transcription factors. Although homeodomain transcription factor cross-repression is an important mechanism for specifying distinct neuronal cell types (Akin and Nazarali, 2005; Nishi et al., 2009), our evidence does not support the idea that Vsx1 and Chx10 function in this manner, since Type 7 bipolar cell identity is not lost following the upregulation of Chx10 in the Vsx1-null retina. Instead of regulating cell specification, the cross-repression of Vsxl and Chx10 in differentiating bipolar cells may be required for regulating the correct terminal gene expression program.

\section{ON signaling defects in Vsx1-deficient mice}

The finding that Vsx1 is expressed in Type 7 ON bipolar cells suggested that it might affect $\mathrm{ON}$ signaling in select populations of ganglion and amacrine cells receiving Type 7 input. Since the ON dendrites of ON-OFF DSGCs costratify with Type 7 ON bipolar cells axon terminals (Fig. 6; Lin and Masland, 2005), it raised the possibility that DSGCs could be selectively affected by $V s x 1$ knock-out. Indeed we found a more sustained ON response as well as reduced directional selectivity in DSGCs in Vsx1-null mice, suggesting that these cells are innervated by Type 7 ON bipolar cells. Future experiments using electron microscopy or trans-synaptic viral tracing methods will be necessary to confirm this synaptic connectivity.

Our finding that Cabp5 is upregulated in Vsx1-null Type 7 bipolar cells is interesting, as it provides a hypothetical explanation for the directional selectivity defects observed in Vsx1-null mice. It has been suggested from previous work that Cabp5 functions as a positive regulator of neurotransmitter release by acting directly on voltage-dependent calcium channels (Rieke et al., 2008). This idea is supported by the finding that Cabp5 directly interacts with the CaMbinding domain of Cav1.2 and colocalizes with Cav1.2 in rod bipolar cells (Rieke et al., 2008). Moreover, cotransfection studies in

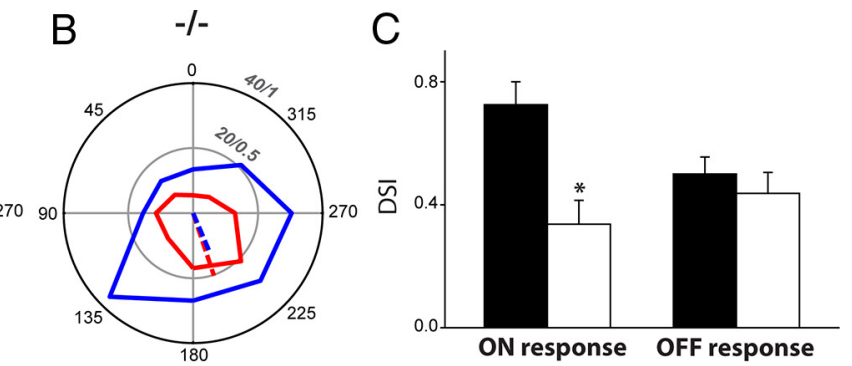

mice. $\boldsymbol{A}, \boldsymbol{B}$, Polar plots representing the spike number (blue for ON spikes; red for OFF spikes) generated by a $400 \mu \mathrm{m}$ spot moving in 8 directions (at $1000 \mu \mathrm{m} / \mathrm{s}$ ) over a wild-type (wt) and a $V s \times 1^{\tau L a C Z / \tau L a C Z}(-/-)$ DSGC, respectively. The dotted lines represent vectors indicating the preferred direction and the amplitude of the directional selectivity index. $\boldsymbol{C}$, The average DSI for the $0 \mathrm{~N}$ response for wild type $(0.72 \pm 0.07 ; n=4)$ and OFF visual signaling directional selectivity

Figure 10. Vsx1 function in bipolar cells. Vsx1 is expressed in 0FF Types 1 and 2 (Chow et al., 2001; Chow et al., 2004) and in 0N Type 7 bipolar cells (this study). In Vsx1-null mice, the expression of six cell type-specific markers in 0FF bipolar cells (recoverin, The 7 bipolar cells (this study). These data reveal a trend in which Vsx 1 functions downstream of cell specification as an activator retinal circuit. While this circuit remains intact in Vsx 1-null mice, a role for Vsx 1 in Type 7 bipolar cell signaling is inferred by defects in directional selective ganglion cell $0 \mathrm{~N}$ response kinetics observed in Vs×1-null mice.

HEK293T cells have shown that Cabp5 can shift the Cav1.2mediated $\mathrm{Ca}^{2+}$ channel activation curve to more positive potentials and suppress calcium-dependent inactivation of the Cav1.2 $\mathrm{Ca}^{2+}$ current (Rieke et al., 2008). As cone bipolar cells also express voltagedependent calcium channels (Pan, 2000), the ectopic expression of Cabp5 seen in Vsx1-null Type 7 bipolar cells may lead to sustained voltage-dependent calcium channel currents (and transmitter release) in this cell and might underlie, in part, the sustained ON flash response of DSGCs observed in Vsx1-null mice. Future work examining the role of Cabp5 and other genes regulated by Vs $x 1$ Type 7 bipolar cells is needed to address this hypothesis.

The more sustained ON response of Vsx1-null DSGCs is reminiscent of early postnatal DSGC responses (Chen et al., 2009). However, there are several differences in the response properties between immature and Vsx1-null DSGS. For example, here we find a decrease in DSI whereas Chen et al. (2009) found that a more sustained response did not alter DSI. In addition, in the immature state, where spike generating mechanisms are not fully developed, DSGCs respond relatively weakly to moving spots compared to mature retina (Elstrott et al., 2008; Chen et al., 2009). In contrast, spike-generating mechanisms in the Vsx1-null mice appear 
to be robust and effectively convert a more sustained input into an increase in spike number. Hence, $V s x 1$ appears to be important for some but not all aspects of maturation of the DSGC circuitry.

The impaired ability of Vsx1-null retina to compute directional selectivity likely arises from alterations in the kinetics of Type 7 bipolar cell synaptic release. As proper encoding of DS requires a fine balance between excitation and inhibition, prolonged bipolar cell output would directly affect the way excitatory and inhibitory inputs are integrated. Our results suggest that prolonged excitatory drive leads to less effective null direction inhibition. In addition, prolonged inputs could also affect the nonlinear dendritic mechanisms that are found to be important in generating DS (Schachter et al., 2010; Trenholm et al., 2011). Thus, a multitude of factors could underlie the compromised ability of Vsx1-null mice to compute DS.

\section{Conclusions}

Collectively, these results represent a significant advancement in our understanding of the role of transcription factors in regulating specific bipolar cell type development and function. Vsxl is necessary for bipolar cell terminal differentiation and is required for the activation and repression of gene expression in OFF and ON bipolar cells, respectively. In turn, $V s x 1$ appears to regulate bipolar cell signaling such that $V s x 1$-null mice exhibit signaling defects in both ON and OFF pathways.

\section{References}

Akin ZN, Nazarali AJ (2005) Hox genes and their candidate downstream targets in the developing central nervous system. Cell Mol Neurobiol 25:697-741.

Bilioni A, Craig G, Hill C, McNeill H (2005) Iroquois transcription factors recognize a unique motif to mediate transcriptional repression in vivo. Proc Natl Acad Sci U S A 102:14671-14676.

Borowska J, Trenholm S, Awatramani GB (2011) An intrinsic neural oscillator in the degenerating mouse retina. J Neurosci 31:5000-5012.

Bramblett DE, Pennesi ME, Wu SM, Tsai MJ (2004) The transcription factor Bhlhb4 is required for rod bipolar cell maturation. Neuron 43:779-793.

Burmeister M, Novak J, Liang MY, Basu S, Ploder L, Hawes NL, Vidgen D, Hoover F, Goldman D, Kalnins VI, Roderick TH, Taylor BA, Hankin MH, McInnes RR (1996) Ocular retardation mouse caused by Chx10 homeobox null allele: impaired retinal progenitor proliferation and bipolar cell differentiation. Nat Genet 12:376-384.

Chen M, Weng S, Deng Q, Xu Z, He S (2009) Physiological properties of direction-selective ganglion cells in early postnatal and adult mouse retina. J Physiol 587:819-828.

Cheng CW, Chow RL, Lebel M, Sakuma R, Cheung HO, Thanabalasingham V, Zhang X, Bruneau BG, Birch DG, Hui CC, McInnes RR, Cheng SH (2005) The Iroquois homeobox gene, Irx5, is required for retinal cone bipolar cell development. Dev Biol 287:48-60.

Chow RL, Snow B, Novak J, Looser J, Freund C, Vidgen D, Ploder L, McInnes RR (2001) Vsx1, a rapidly evolving paired-like homeobox gene expressed in cone bipolar cells. Mech Dev 109:315-322.

Chow RL, Volgyi B, Szilard RK, Ng D, McKerlie C, Bloomfield SA, Birch DG, McInnes RR (2004) Control of late off-center cone bipolar cell differentiation and visual signaling by the homeobox gene Vsxl. Proc Natl Acad Sci U S A 101:1754-1759.

Clark AM, Yun S, Veien ES, Wu YY, Chow RL, Dorsky RI, Levine EM (2008) Negative regulation of Vsx 1 by its paralog Chx10/Vsx2 is conserved in the vertebrate retina. Brain Res 1192:99-113.

Coombs J, van der List D, Wang GY, Chalupa LM (2006) Morphological properties of mouse retinal ganglion cells. Neuroscience 140:123-136.

Dorval KM, Bobechko BP, Ahmad KF, Bremner R (2005) Transcriptional activity of the paired-like homeodomain proteins CHX10 and VSX1. J Biol Chem 280:10100-10108.

Elshatory Y, Gan L (2008) The LIM-homeobox gene Islet-1 is required for the development of restricted forebrain cholinergic neurons. J Neurosci 28:3291-3297.

Elstrott J, Anishchenko A, Greschner M, Sher A, Litke AM, Chichilnisky EJ, Feller
MB (2008) Direction selectivity in the retina is established independent of visual experience and cholinergic retinal waves. Neuron 58:499-506.

Feng L, Xie X, Joshi PS, Yang Z, Shibasaki K, Chow RL, Gan L (2006) Requirement for Bhlhb5 in the specification of amacrine and cone bipolar subtypes in mouse retina. Development 133:4815-4825.

Fried SI, Münch TA, Werblin FS (2002) Mechanisms and circuitry underlying directional selectivity in the retina. Nature 420:411-414.

Ghosh KK, Bujan S, Haverkamp S, Feigenspan A, Wässle H (2004) Types of bipolar cells in the mouse retina. J Comp Neurol 469:70-82.

Haeseleer F, Sokal I, Verlinde CL, Erdjument-Bromage H, Tempst P, Pronin AN, Benovic JL, Fariss RN, Palczewski K (2000) Five members of a novel $\mathrm{Ca}^{2+}$-binding protein (CABP) subfamily with similarity to calmodulin. J Biol Chem 275:1247-1260.

Haverkamp S, Ghosh KK, Hirano AA, Wässle H (2003) Immunocytochemical description of five bipolar cell types of the mouse retina. J Comp Neurol 455:463-476.

Huang L, Shanker YG, Dubauskaite J, Zheng JZ, Yan W, Rosenzweig S, Spielman AI, Max M, Margolskee RF (1999) Ggamma13 colocalizes with gustducin in taste receptor cells and mediates IP3 responses to bitter denatonium. Nat Neurosci 2:1055-1062.

Huang L, Max M, Margolskee RF, Su H, Masland RH, Euler T (2003) G protein subunit $\mathrm{G}$ gamma 13 is coexpressed with $\mathrm{G}$ alpha $\mathrm{o}$, $\mathrm{G}$ beta 3 , and G beta 4 in retinal ON bipolar cells. J Comp Neurol 455:1-10.

Kerschensteiner D, Liu H, Cheng CW, Demas J, Cheng SH, Hui CC, Chow RL, Wong RO (2008) Genetic control of circuit function: Vsx1 and Irx5 transcription factors regulate contrast adaptation in the mouse retina. J Neurosci 28:2342-2352.

Koizumi A, Jakobs TC, Masland RH (2011) Regular mosaic of synaptic contacts among three retinal neurons. J Comp Neurol 519:341-357.

Lin B, Masland RH (2005) Synaptic contacts between an identified type of ON cone bipolar cell and ganglion cells in the mouse retina. Eur J Neurosci 21:1257-1270.

Masland RH (2001) The fundamental plan of the retina. Nat Neurosci $4: 877-886$

Mataruga A, Kremmer E, Müller F (2007) Type 3a and type 3b OFF cone bipolar cells provide for the alternative rod pathway in the mouse retina. J Comp Neurol 502:1123-1137.

Nishi Y, Ji H, Wong WH, McMahon AP, Vokes SA (2009) Modeling the spatio-temporal network that drives patterning in the vertebrate central nervous system. Biochim Biophys Acta 1789:299-305.

Ohtoshi A, Wang SW, Maeda H, Saszik SM, Frishman LJ, Klein WH, Behringer RR (2004) Regulation of retinal cone bipolar cell differentiation and photopic vision by the CVC homeobox gene Vsx1. Curr Biol 14:530-536.

Pan ZH (2000) Differential expression of high- and two types of lowvoltage-activated calcium currents in rod and cone bipolar cells of the rat retina. J Neurophysiol 83:513-527.

Reményi A, Schöler HR, Wilmanns M (2004) Combinatorial control of gene expression. Nat Struct Mol Biol 11:812-815.

Rieke F, Lee A, Haeseleer F (2008) Characterization of $\mathrm{Ca}^{2+}$-binding protein 5 knockout mouse retina. Invest Ophthalmol Vis Sci 49:5126-5135.

Schachter MJ, Oesch N, Smith RG, Taylor WR (2010) Dendritic spikes amplify the synaptic signal to enhance detection of motion in a simulation of the direction-selective ganglion cell. PLoS Comput Biol 6:pii:e1000899.

Shi Z, Jervis D, Nickerson PE, Chow RL (2011) A requirement for the paired-like homeodomain transcription factor Vsx1 in Type 3a mouse retinal bipolar cell terminal differentiation. J Comp Neurol. Advance online publication. Retrieved June 14, 2011. doi:10.1002/cne.22697.

Svingen T, Tonissen KF (2006) Hox transcription factors and their elusive mammalian gene targets. Heredity 97:88-96.

Taylor WR, Vaney DI (2002) Diverse synaptic mechanisms generate direction selectivity in the rabbit retina. J Neurosci 22:7712-7720.

Trenholm S, Johnson K, Li X, Smith RG, Awatramani GB (2011) Parallel mechanisms encode direction in the retina. Neuron 71:683-694.

Wässle H, Puller C, Müller F, Haverkamp S (2009) Cone contacts, mosaics, and territories of bipolar cells in the mouse retina. J Neurosci 29:106-117.

Wolberger C (1999) Multiprotein-DNA complexes in transcriptional regulation. Annu Rev Biophys Biomol Struct 28:29-56.

Xu ZP, Dutra A, Stellrecht CM, Wu C, Piatigorsky J, Saunders GF (2002) Functional and structural characterization of the human gene BHLHB5, encoding a basic helix-loop-helix transcription factor. Genomics 80:311-318. 